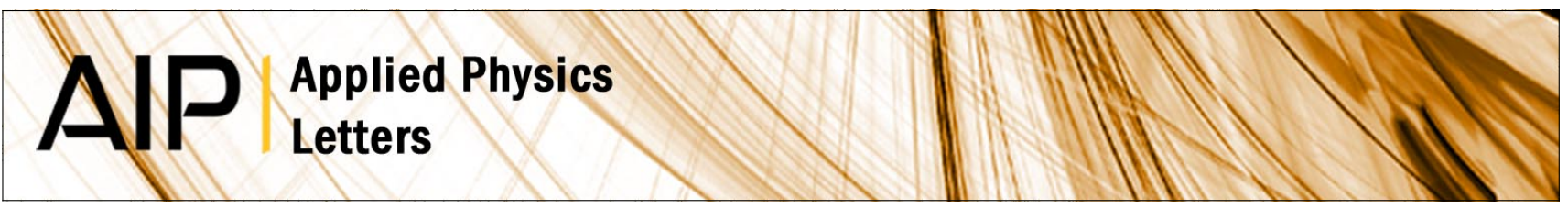

\title{
Assembly of microparticles by optical trapping with a photonic crystal nanocavity
}

C. Renaut, J. Dellinger, B. Cluzel, T. Honegger, D. Peyrade et al.

Citation: Appl. Phys. Lett. 100, 101103 (2012); doi: 10.1063/1.3692104

View online: http://dx.doi.org/10.1063/1.3692104

View Table of Contents: http://apl.aip.org/resource/1/APPLAB/v100/i10

Published by the American Institute of Physics.

\section{Related Articles}

Light trapping in solar cells: When does a Lambertian scatterer scatter Lambertianly?

J. Appl. Phys. 112, 094504 (2012)

Video-based and interference-free axial force detection and analysis for optical tweezers

Rev. Sci. Instrum. 83, 103704 (2012)

Practical axial optical trapping

Rev. Sci. Instrum. 83, 103106 (2012)

Photophoretic trampoline-Interaction of single airborne absorbing droplets with light Appl. Phys. Lett. 101, 131115 (2012)

Auto- and cross-power spectral analysis of dual trap optical tweezer experiments using Bayesian inference Rev. Sci. Instrum. 83, 095116 (2012)

\section{Additional information on Appl. Phys. Lett.}

Journal Homepage: http://apl.aip.org/

Journal Information: http://apl.aip.org/about/about_the_journal

Top downloads: http://apl.aip.org/features/most_downloaded

Information for Authors: http://apl.aip.org/authors

\section{ADVERTISEMENT}
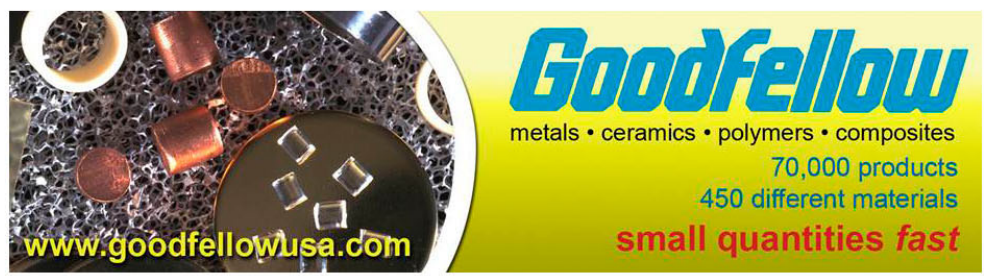


\title{
Assembly of microparticles by optical trapping with a photonic crystal nanocavity
}

\author{
C. Renaut, ${ }^{1,2,3, a)}$ J. Dellinger, ${ }^{1}$ B. Cluzel, ${ }^{1, b)}$ T. Honegger, ${ }^{3}$ D. Peyrade, ${ }^{3}$ E. Picard, ${ }^{2}$ \\ F. de Fornel, ${ }^{1}$ and E. Hadji ${ }^{2}$ \\ ${ }^{1}$ Groupe d'Optique de Champ Proche - LRC CEA nDSM-08-36, Laboratoire Interdisciplinaire Carnot de \\ Bourgogne, UMR CNRS n6303, Université de Bourgogne, Dijon, France \\ ${ }^{2}$ Laboratoire Silicium Nanoélectronique Photonique et Structures, INAC/SP2M/SiNaPS, CEA Grenoble, \\ France \\ ${ }^{3}$ CNRS/UJF-Grenoble1/CEA LTM, 17 avenue des Martyrs, 38054 Grenoble, France
}

(Received 29 November 2011; accepted 8 February 2012; published online 5 March 2012)

\begin{abstract}
In this work, we report the auto-assembly experiments of micrometer sized particles by optical trapping in the evanescent field of a photonic crystal nanocavity. The nanocavity is inserted inside an optofluidic cell designed to enable the real time control of the nanoresonator transmittance as well as the real time visualization of the particles motion in the vicinity of the nanocavity. It is demonstrated that the optical trap above the cavity enables the assembly of multiple particles in respect of different stable conformations. (C) 2012 American Institute of Physics. [http://dx.doi.org/10.1063/1.3692104]
\end{abstract}

Optical cavities miniaturized at the submicrometer scale $^{1}$ enable both spatial and spectral confinement of an electromagnetic field at the nanoscale. The resulting field localization inside the resonator optical near-field leads to strong optical forces which are expected to be of a great interest for optical handling of nanoparticles. As a consequence, the integration of these resonators on an optofluidic chip promises future exciting applications in the field of life science for nanoparticles ${ }^{2}$ or biological elements ${ }^{3}$ sensing, trapping, and sorting. In this context, recent proof-on-concept experiments of micro and nanoparticles trapping by a ring resonator ${ }^{4}$ or a photonic crystal nanocavity ${ }^{5}$ have been reported. In this work, we report the observations of particles trapping and auto-assembly inside the evanescent field of a photonic crystal nanocavity integrated within a silicon-on-insulator (SOI) optofluidic chip and operating at telecommunication wavelength.

The SOI photonic chip fabricated by electron beam lithography plus inductively coupled plasma etching on a $200 \mathrm{~mm}$ SOI wafer consists in high quality factor and small modal volume $\left(\mathrm{V} \sim(\lambda / \mathrm{n})^{3}\right)$ cavity integrated on a $500 \mathrm{~nm}$ wide ridge waveguide. The cavities are defined by two photonic crystal (PC) tapered mirrors designed to improve the quality factor $(\mathrm{Q})$ inside the cavity volume $(\mathrm{V})^{6}$ such cavities achieve $\mathrm{Q} / \mathrm{V}$ factors, which rival the best $2 \mathrm{D}$ PC cavities. ${ }^{7,8}$ The cavity designed in this work operates in a moderate $\mathrm{Q}$ regime $(Q \sim 2000)$ in order to minimize the near-field perturbation of the cavity resonances by the presence of a nanometric object in their vicinity. ${ }^{9}$ The standing wave pattern of the cavity is composed of several intensity maxima each forming equally probable single particle trapping site. ${ }^{10} \mathrm{~A}$ scanning electron microscope (SEM) view of a typical nanocavity is shown in Fig. 1(a). The center of the nanocavity is pointed by a triangle shaped mark in order to enable a clear visualization of the nanocavity's position for the optical trap-

\footnotetext{
${ }^{a}$ Electronic mail: claude.renaut@u-bourgogne.fr.

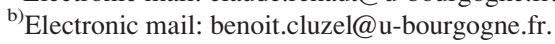

ping experiments presented hereafter. A polydimethylsiloxane (PDMS) layer micropatterned by a circular aperture is then aligned and bonded onto the SOI chip. ${ }^{11}$ The nanocavities are centered on the PDMS circular apertures, and the resulting cell is filled with a $4 \mu \mathrm{L}$ volume of green fluorescent polystyrene (PS) microbeads ( $1 \mu \mathrm{m}$ diameter) solution with a concentration of $3.88 \times 10^{8}$ part. $\mathrm{mL}^{-1}$. The cells are finally sealed with a microscope cover slip as shown in Fig. 1(b).

Then, the optical characterization is performed in farfield by using an endfire coupling scheme. ${ }^{12}$ An amplified transverse electrical (TE) polarized tunable laser source is coupled to the input waveguide, thanks to a polished polarization maintained optical fiber tip. The light coming from the waveguide after passing through the nanocavity is collected, thanks to an objective, and detected by an InGaAs photodetector. The lightened nanocavities at the center of the cell are observed simultaneously, thanks to a microscope operating with a dual illumination. The green fluorescence of the PS particles under blue light excitation is visualized, thanks to a classical fluorescent microscopy scheme while a grazing incidence red illumination enables the simultaneous observation of the nanocavity and its triangle. In this configuration, the experimental setup enables a synchronous
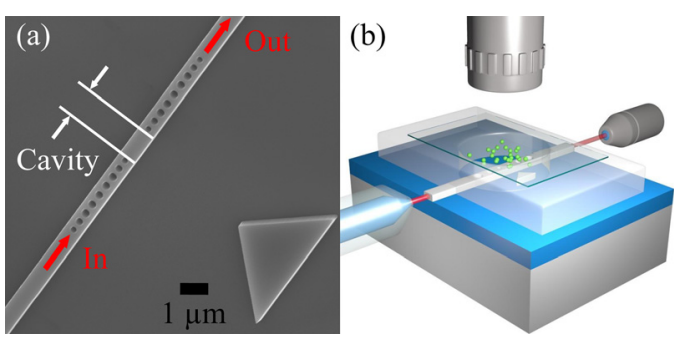

FIG. 1. (Color online) (a) Scanning electron microscope view of the nanocavity resonator made of silicon on insulator substrate. The view includes the rib waveguides for light input and output. (b) Schematic view of the optofluidic cell embedding the nanocavity and the polystyrene microbeads solution. 


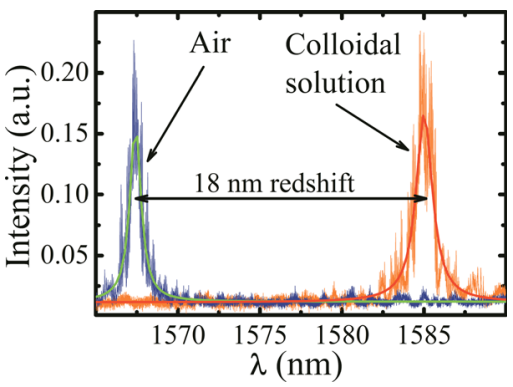

FIG. 2. (Color online) Optical far field spectroscopy of the nanocavity in air and in the optofluidic cell filled with the microbeads solution. The nanoresonator exhibits a single resonance red shifted in the optofluidic cell.

optical control of the nanoresonator transmittance and of the particles motion in the vicinity of the nanocavity.

As plotted in Fig. 2, the immerged nanocavity exhibits a single resonance redshifted compared with the one measured without colloidal solution. The measured $18 \mathrm{~nm}$ redshift value is associated to the change of the optical index of the media surrounding the SOI nanocavity, which indicates that all the cavity holes are filled with the aqueous solution. As shown on the figure, the cavity transmittance at resonance remains unchanged while the cavity Q-factor is slightly reduced to 1600 . This decrease is mainly due to a slight decrease of the photonic crystal mirrors reflectivity due to a lower index contrast between silicon and water compared to that one between silicon and air in the case of the unfilled cavity.

As observed experimentally and visible on the media file available online related to Fig. 3, a stable optical trap suppressing the particle motion is obtained for injected power higher than $300 \mu \mathrm{W}$. Selected snapshots of the video are reported in Figure 3 which illustrates the particle diffusion far away from the nanocavity (Figs. 3(a) and 3(b)), the
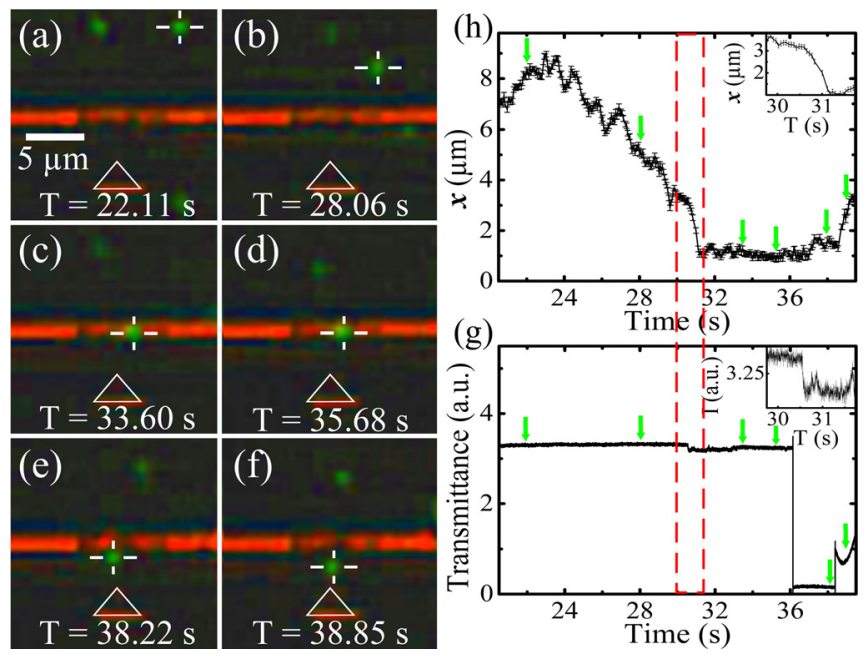

FIG. 3. (Color online) Snapshots of the related media file illustrate (a) and (b) the particle diffusion far away from the nanocavity, (c) and (d) the particle trapping, and (e) and (f) the particle release as soon as the laser is switched off. (g) Particle motion measurement, we tracked the relative position $x$ of the trapped particle compared to the cavity center as a function of time and compared it with the cavity transmittance measurements in (h). (h) Nanocavity far-field optical transmittance recorded synchronously with the particles motion over the resonator (enhanced online) [URL: http:// dx.doi.org/10.1063/1.3692104.1]. particle trapping (Figs. 3(c) and 3(d)), and the particle release as soon as the laser is switched off (Figs. 3(e) and 3(f)). From the particle motion measurement, we tracked the relative position of the trapped particle compared to the cavity center as a function of time (Fig. 3(g)) and compared it to the cavity transmittance measurements (Fig. 3(h)). As highlighted by the inset on the figure, the particle trapping is associated with a slight decrease of the cavity transmittance corresponding to the resonance perturbation due to the particle-cavity near-field interactions. We note here that this decay is negligible for the reported trapping experiments with a moderate Q-factor cavity. It can be noticed that the trapping position of the particle does not match the cavity center, the trapping site being associated to one of the maxima of the electric field intensity distribution inside the nanocavity.

Finally, as presented in the second mediafile related to Fig. 4, multiple particles trapping is also observed in the nanocavity optical near-field. Remarkably, in all the experiments involving several particles, we always observed their auto-assembly according to well defined conformations in the vicinity of the nanocavity. The stable conformations
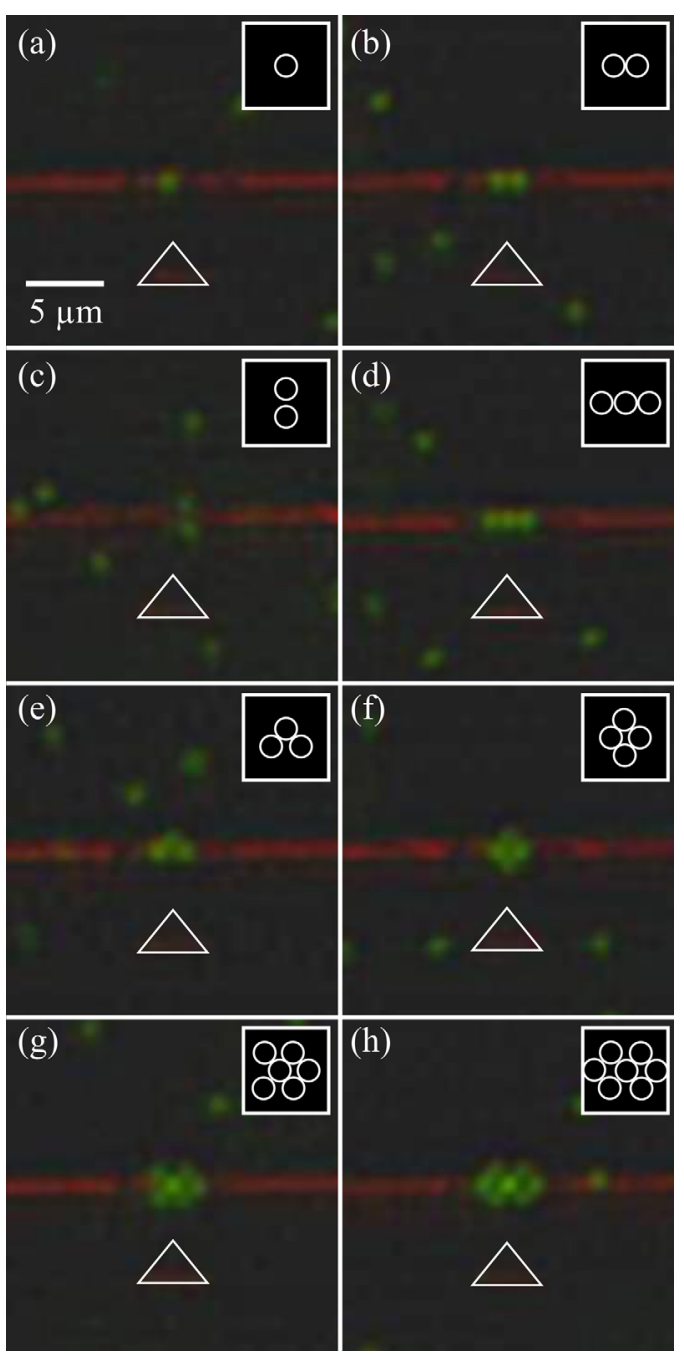

FIG. 4. (Color online) The stable conformations inside the optical trap, visible online on the media file, obtained with (a) 1 particle, (b) and (c) 2 particles, (d) and (e) 3 particles, (f) 4 particles, (g) 6 particles, and (h) 7 particles (enhanced online) [URL: http://dx.doi.org/10.1063/1.3692104.2]. 
inside the optical trap are obtained for one, two, three, four, six, and seven particles. On the figure, we note that the stables particles organizations follow very simple geometrics structures, straight lines, triangles, rhombuses, and combinations of these basic shapes. The corresponding conformations are reported in Fig. 4. As shown on the associated mediafile, we observed that the conformation of the particles changes dynamically as soon as another particle penetrates the cavity optical near-field, switching from a stable conformation with $\mathrm{N}$ particles to another one with $(\mathrm{N}+1)$ particles. This is particularly obvious on the video for the conformal change between 3 and 4 trapped particles. These stable conformations result from the resonant mode electromagnetic field distribution within the nanocavity volume and from its near-field interactions with the particles. As long as these near-field interactions remain weak, i.e., that the cavity transmittance remains unchanged with or without particle, the number of trapped particles above the nanocavity can be increased only according to the stable conformations. This implies that the particles cannot be released independently. The cavity optical forces allow the self-assembly of multiple particles without any self induced back action (SIBA) $)^{13,14}$ process since the cavity-particles interaction remains weak. However, a clever use of the near-field interactions between the particles and a higher Q-factor nanocavity could benefit from a SIBA optical trapping. This could enable a full control of the stability or the instability of the conformations assembly as well as a dynamical control of the transitions between them.

In conclusion, we have reported the optical trapping and assembly experiments of microparticles by a photonic crystal nanocavity integrated to a silicon-on-insulator optofluidic chip operating at telecommunication wavelengths. The fabricated structures designed to enable the real time control of the nanoresonator transmittance as well as the real time visualization of the particles motion in the vicinity of the nano- cavity are characterized optically. We have shown that an injected power larger than $300 \mu \mathrm{W}$ leads to the formation of a stable optical trap above the nanocavity. Moreover, we have observed that the trapped particles are arranged above the cavity in respect to different stable conformations. The results reported here offer promising perspectives to achieve on-chip active control of particles assembly and therefore develop configurable optofluidics devices. Taking advantages of air-slotted geometries ${ }^{15,16}$ to further expand the nanoparticles dispatching and localization capabilities can now be envisioned.

${ }^{1}$ K. J. Vahala, Nature 424, 839 (2003).

${ }^{2}$ D. Erickson, X. Serey, Y. F. Chen, and S. Mandal, Lab Chip 11, 995 (2011).

${ }^{3}$ M. Righini, P. Ghenuche, S. Cherukulappurath, V. Myroshnychenko, F. J. Garcia de Abajo, and R. Quidant, Nano Lett. 9, 3387 (2009).

${ }^{4}$ S. Lin, E. Schonbrun, and K. Crozier, Nano Lett. 10, 2408 (2010).

${ }^{5}$ S. Mandal, X. Serey, and D. Erickson, Nano Lett. 10, 99 (2010).

${ }^{6}$ P. Velha, J. C. Rodier, P. Lalanne, J. P. Hugonin, D. Peyrade, E. Picard, T. Charvolin, and E. Hadji, Appl. Phys. Lett. 89, 171121 (2006).

${ }^{7}$ A. R. M. Zain, N. P. Johnson, M. Sorel, and R. De La Rue, Opt. Express 16, 12084 (2008).

${ }^{8}$ M. Notomi, E. Kuramochi, and T. Tanabe, Nature Photon. 2, 741 (2008).

${ }^{9}$ B. Cluzel, L. Lalouat, P. Velha, E. Picard, D. Peyrade, J. C. Rodier, T. Charvolin, P. Lalanne, F. de Fornel, and E. Hadji, Opt. Express 16, 279 (2008).

${ }^{10}$ L. Lalouat, B. Cluzel, F. de Fornel, P. Velha, P. Lalanne, D. Peyrade, E. Picard, T. Charvolin, and E. Hadji, Appl. Phys. Lett. 92, 111111 (2008).

${ }^{11}$ T. Honegger, K. Berton, T. Pinedo-Rivera, and D. Peyrade, Microelectron. Eng. 86, 1401 (2009).

${ }^{12}$ M. Zelsmann, E. Picard, T. Charvolin, E. Hadji, B. Dal Zotto, M. E. Nier, C. Seassal, P. Rojo-Romeo, and X. Letartre, Appl. Phys. Lett. 81, 2340 (2002).

${ }^{13}$ M. L. Juan, R. Gordon, Y. Pang, F. Eftekhari, and R. Quidant, Nat. Phys. 5, 915 (2009).

${ }^{14}$ M. L. Juan, M. Righini, and R. Quidant, Nature Photon. 5, 349 (2011).

${ }^{15}$ K. Foubert, L. Lalouat, B. Cluzel, E. Picard, D. Peyrade, E. Delamadeleine, F. de Fornel, and E. Hadji, Appl. Phys. Lett. 93, 251103 (2008).

${ }^{16}$ K. Foubert, L. Lalouat, B. Cluzel, E. Picard, D. Peyrade, F. de Fornel, and E. Hadji, Appl. Phys. Lett. 94, 251111 (2009). 